\title{
Analysis of the longitudinal space charge impedance of a round uniform beam inside parallel plates and rectangular chambers
}

\author{
L. Wang* \\ SLAC National Accelerator Laboratory, Menlo Park, California 94025, USA \\ Y. Li \\ Department of Physics, Michigan State University, East Lansing, Michigan 48824, USA
}

(Received 10 July 2014; published 3 February 2015)

\begin{abstract}
This paper analyzes the longitudinal space charge impedances of a round uniform beam inside a rectangular and parallel plate chambers using the image charge method. This analysis is valid for arbitrary wavelengths, and the calculations converge rapidly. The research shows that only a few of the image beams are needed to obtain a relative error less than $0.1 \%$. The beam offset effect is also discussed in the analysis.

DOI: 10.1103/PhysRevSTAB.18.024201

PACS numbers: 29.27.Bd, 29.20.db
\end{abstract}

\section{INTRODUCTION}

The longitudinal space charge (LSC) impedances are important factors that should be taken into account in the design and operation of modern particle accelerators, especially the ones with low energy and high beam intensities, which are prone to microwave instabilities induced by the LSC fields [1,2]. This is also important for the free-electron lasers (FELs), where the electron's energy is up to a few $\mathrm{GeV}$ but its peak current is in the order of kA [3]. Strong microbunching of the order of micrometers has been recently observed [4] in Linac Coherent Light Source (LCLS) [5] at SLAC National Accelerator Laboratory (SLAC). On the other hand, the LSC, as a positive factor, increases the current modulation and can be used as an amplifier for FEL [6]. Various LSC field models have been proposed and studied extensively by different methods in the existing literature, such as Refs. [7-22]. The LSC impedances in the entire wavelength spectrum of a beam, in free space [8] and inside a round chamber [13-16], have been intensively studied. The LSC impedance of a beam inside a round chamber in the longwavelength limit is also well known [17-21].

In many accelerators, the configurations of the real beamchamber system can be simplified as a field model consisting of a round beam moving inside a rectangular chamber. When the aspect ratio of the cross section of the rectangular chamber is large, it can be simplified further as a pair of parallel plates [23]. If the beam has longitudinal density modulation, due to mismatch of the cross-sectional shapes between the beam and chamber, the exact closed-form

\footnotetext{
*WANGLF@slac.stanford.edu

Published by the American Physical Society under the terms of the Creative Commons Attribution 3.0 License. Further distribution of this work must maintain attribution to the author $(s)$ and the published article's title, journal citation, and DOI.
}

solutions to the three-dimensional (3D) space charge fields and the corresponding LSC impedances of the above two cases cannot be obtained by the method of separation of variables. Until now, few works were carried out for obtaining the LSC impedances in the entire wavelength spectrum of the field models in a rectangular chamber and between a pair of parallel plates. Though Ref. [7] used the method of conformal mapping and Faraday's law applied to a rectangular integral loop to derive the analytical LSC fields and impedances of a round beam in rectangular chamber, it neglected the 3D field effect when the density perturbation wavelength is small. Hence, this method and results are only valid in the long-wavelength limit and are not appropriate for the study of microwave or microbunching instabilities. Since in most accelerators the ratios of the transverse chamber dimensions to the beam diameters are large, Ref. [12] provides the approximate solutions to the field models of a round beam with planar and rectangular boundary conditions, assuming the 3D image charge fields of the round beam can be approximated by the image fields of a line charge. The resulting LSC fields and impedances are valid for the whole perturbation wavelength spectrum. When the ratio of the beam diameter to the transverse chamber dimension approaches unity, the relative errors of the approximated LSC impedances will become larger. In addition, the LSC field of a round beam between parallel plates has been studied in Ref. [22], but the results are only valid in the long-wavelength limit.

This paper proposes an image charge method to calculate the LSC impedances of a round beam between parallel plates and inside a rectangular chamber. It is well known that the solutions to the LSC fields of a round transversely uniform beam with sinusoidal line charge density modulation in free space are available in a closed form [8]. If the beam were placed inside a rectangular chamber or between parallel plates, due to planar symmetry and mirroring, the associated total image charge fields of the infinite chain 
(parallel plates model) or grid (rectangular chamber model) of the image beams can be calculated by simple summation. Adding the self-fields of the round beam in free space, the total LSC fields and impedances of the two models in discussion can be obtained. Through case study, we found the calculated LSC fields and impedances converge fairly quickly with the number of image beams. Usually only a finite and small number of image beams are needed to obtain the LSC fields and impedances with relative errors less than $0.1 \%$. The resolution of the calculated LSC fields and impedances depends on the number of image beams used in the calculation, rather than the ratio of the beam diameter to the transverse chamber dimension, as was the case in Ref. [12].

The image charge method is one of the popular methods in the study of the charged particle field, especially for solving the Poisson's equation (i.e., Ref. [24]). In some literature, the round beam is approximated by a line charge $[12,20]$ in calculation of the image charge field for simplicity. In this paper, the exact image beams are included in the calculation of the full-spectrum LSC impedance.

This paper is organized as follows. Section II briefly introduces the wave equations describing the space charge fields of the charged beam. Section III briefly introduces the numerical calculation method for general geometries of the beam and beam pipe. Section IV provides a short review for the LSC fields and impedances of a round beam, in free space and inside a round chamber, respectively. Sections V and VI respectively calculate the LSC impedances of a round beam, between parallel plates and inside a rectangular chamber, using the image charge method.

\section{WAVE EQUATIONS}

The wave equation describing the electric field $\boldsymbol{E}$ is

$$
\nabla^{2} \boldsymbol{E}-\frac{1}{c^{2}} \frac{\partial \boldsymbol{E}}{\partial t}=\frac{\nabla \rho}{\varepsilon_{0}}+\mu_{0} \frac{\partial \boldsymbol{J}}{\partial t},
$$

where $\epsilon_{0}=8.85 \times 10^{-12} \mathrm{~F} / \mathrm{m}$ and $\mu_{0}=4 \pi \times 10^{-7} \mathrm{~N} / \mathrm{A}^{2}$ are the permittivity and permeability of free space, respectively; $c=\frac{1}{\sqrt{\varepsilon_{0} \mu_{0}}}=3 \times 10^{8} \mathrm{~m} / \mathrm{s}$ is the speed of light in free space; $\rho$ and $\boldsymbol{J}$ are the charge and the current densities, respectively, they obey the following continuity equation:

$$
\frac{\partial \rho}{\partial t}+\vec{\nabla} \cdot \boldsymbol{J}=0
$$

Assuming that the beam is moving with a constant longitudinal speed $\boldsymbol{v}=\beta c \hat{z}$ along the $z$ axis, where $\beta$ is the relativistic speed, $\hat{z}$ is the unit vector of the longitudinal coordinate, then $\boldsymbol{J}$ can be expressed as $\boldsymbol{J}=\boldsymbol{J} \hat{z}$. For a perturbed beam, its volume charge density $\rho$ and current density $J$ consist of unperturbed $(\mathrm{dc})$ components and perturbed higher order harmonic components. Since the physics of the unperturbed components is trivial and does not contribute to the LSC field, we only need to focus on the physics associated with those higher order harmonic components. We will study one particular harmonic component with a frequency $\omega$ (or wave number $k$ ) in the rest of this paper and omit the subscript $k$ in the variables of fields, density and current for simplicity. The single harmonic component of the charge density, current density and beam current can be expressed using the wave assumption:

$$
\begin{gathered}
\rho(x, y, z, t)=\lambda_{z} \rho_{\perp}(x, y) e^{-i \omega(t-z / v)}, \\
\boldsymbol{J}=\rho \boldsymbol{v}=\hat{z} \beta c \lambda_{z} \rho_{\perp}(x, y) e^{-i \omega(t-z / v)}, \\
I=\lambda_{z} v e^{-i \omega(t-z / v)}=\bar{I} e^{-i \omega(t-z / v)},
\end{gathered}
$$

where $\lambda_{z}$ is the magnitude of the harmonic line charge density, $\rho_{\perp}$ is the transverse beam distribution function normalized by $\int \rho_{\perp}(x, y) d x d y=1$. We will work with only the $z$ components of the vectors $\boldsymbol{E}, \boldsymbol{J}$ and $\nabla$. Hence, the differentials of the longitudinal harmonic components of $J$ and $\rho$ can be expressed as

$$
\begin{gathered}
\mu_{0} \frac{\partial J_{z}}{\partial t}=-i \mu_{0} \omega v \lambda_{z} \rho_{\perp} e^{-i \omega\left(t-\frac{z}{v}\right)}, \\
\frac{1}{\varepsilon_{0}} \frac{\partial \rho}{\partial z}=\frac{i \omega \lambda_{z}}{\varepsilon_{0} v} \rho_{\perp} e^{-i \omega(t-z / v)} .
\end{gathered}
$$

The $z$ component of the harmonic electric field can be written as

$$
\mathbf{E}_{z}(x, y, z, t)=\hat{\mathbf{z}} \mathrm{E}_{z}(k, x, y) e^{-i \omega(t-z / v)} .
$$

Substituting Eqs. (4)-(6) into Eq. (1), the amplitude of the longitudinal electric field $E_{z}(k, x, y)$ satisfies the following equation:

$$
\left(\nabla_{\perp}^{2}-\frac{k^{2}}{\gamma^{2}}\right) \mathrm{E}_{z}=i \frac{k \lambda_{z}}{\varepsilon_{0} \gamma^{2}} \rho_{\perp}(x, y),
$$

where $\nabla_{\perp}^{2}=\frac{\partial^{2}}{\partial x^{2}}+\frac{\partial^{2}}{\partial y^{2}}, k=\frac{\omega}{v}=\frac{\omega}{\beta c}$ and $\frac{1}{\gamma^{2}}=1-\frac{v^{2}}{c^{2}}=1-\beta^{2}$.

The LSC impedance per unit length of an accelerator with circumference $L$ at an arbitrary transverse coordinate $(x, y)$ is defined as

$$
\begin{gathered}
\frac{Z_{\|}(k, x, y)}{L}=-\frac{E_{z}(k, x, y)}{\overline{\mathrm{I}}}=-\frac{E_{z}(k, x, y)}{\lambda_{z} \beta c} . \\
\text { III. FEM SIMULATION FOR } \\
\text { ARBITRARY GEOMETRY }
\end{gathered}
$$

Equation (7) with arbitrary cross-sectional geometries of the beam and beam pipe can be solved numerically using the finite element method (FEM) [25]. The FEM equation is 


$$
\left(\mathbf{M}+\frac{k^{2}}{\gamma^{2}} \mathbf{B}\right) \mathrm{E}_{z}=\mathbf{Q}
$$

with

$$
\begin{gathered}
M_{m, n}^{e}=\iint_{S^{e}}\left(\frac{\partial N_{m}}{\partial x} \frac{\partial N_{n}}{\partial x}+\frac{\partial N_{m}}{\partial y} \frac{\partial N_{n}}{\partial y}\right) d x d y, \\
B_{m, n}^{e}=\iint_{S^{e}} N_{m} N_{n} d x d y \\
Q_{m}^{e}=-i \frac{k q_{m}}{\varepsilon_{0} \gamma^{2}} .
\end{gathered}
$$

Here $\boldsymbol{M}$ is the stiffness matrix with element $M_{m, n}^{e}$ ( $m$ and $n$ are the node indexes of the finite element). $S^{e}$ is the integration boundary of the finite element. $N(x, y)$ is called the shape function (similar to the weighting factor) in FEM, by which the fields at a field point $P(x, y)$ within an element can be interpolated by the fields of its neighboring nodes. It is related to the coordinates of the field point $P(x, y)$ and the nodes of the element region. $q_{m}$ is the charge at the node $m$, which is proportional to $\lambda_{z}$. The current $I$ has the similar dependence on $\lambda_{z}$. Therefore, the LSC impedance given by Eq. (8) is independent of $\lambda_{z}$ as expected. The $E_{z}$ of Eq. (9) at all nodes satisfying Eqs. (9)-(12) and the boundary condition $E_{z}=0$ on the chamber wall can be solved numerically. Then the corresponding longitudinal space charge impedances can be calculated using Eq. (8).

\section{IN FREE SPACE AND INSIDE A ROUND CHAMBER}

For an infinitely long round beam with uniform transverse density within beam radius $a$, its transverse density distribution function is

$$
\rho_{\perp}(r)= \begin{cases}\frac{1}{\pi a^{2}} & (r \leq a) \\ 0 & (r>a),\end{cases}
$$

where $r^{2}=x^{2}+y^{2}$. The general solution of Eq. (7) with the above beam distribution is [26]

$$
E_{Z}(k, r)= \begin{cases}A_{1} I_{0}\left(\frac{k r}{\gamma}\right)+A_{2} K_{0}\left(\frac{k r}{\gamma}\right) & r>a, \\ A_{3} I_{0}\left(\frac{k r}{\gamma}\right)-i \frac{\lambda_{z}}{k \pi a^{2} \varepsilon_{0}} & r \leq a,\end{cases}
$$

where $I_{0}(x)$ and $K_{0}(x)$ are the zeroth order modified Bessel functions of the first and second kinds, respectively. In free space, the field strength at $r \rightarrow \infty$ should be finite. Therefore $A_{1}=0$. The continuity conditions of the field and its derivative at $r=a$ give [8]

$E_{z}^{\mathrm{free}}(k, r)= \begin{cases}-i \frac{\lambda_{z}}{k \pi a^{2} \varepsilon_{0}}\left[1-\frac{k a}{\gamma} K_{1}\left(\frac{k a}{\gamma}\right) I_{0}\left(\frac{k r}{\gamma}\right)\right] & (r \leq a), \\ -i \frac{\lambda_{z}}{k \pi a^{2} \varepsilon_{0}}\left[\frac{k a}{\gamma} K_{0}\left(\frac{k r}{\gamma}\right) I_{1}\left(\frac{k a}{\gamma}\right)\right] & (r>a),\end{cases}$

where $I_{1}(x)$ and $K_{1}(x)$ are the first order modified Bessel functions of the first and second kinds, respectively.

The superscript "free" on the left hand stands for "free space." With the property of $I_{0}(0)=1$, Eqs. (8) and (15) yield the LSC field and impedance per unit length on the beam axis $(r=0)$ as

$$
\begin{gathered}
E_{z}^{\mathrm{free}}(k, 0)=-i \frac{\lambda_{z}}{k \pi a^{2} \varepsilon_{0}}\left[1-\frac{k a}{\gamma} K_{1}\left(\frac{k a}{\gamma}\right)\right], \\
\frac{Z_{\|}^{\mathrm{free}}(k, 0)}{L}=i \frac{Z_{0}}{k \pi a^{2} \beta}\left[1-\frac{k a}{\gamma} K_{1}\left(\frac{k a}{\gamma}\right)\right],
\end{gathered}
$$

where $Z_{0}=1 / \varepsilon_{0} c \approx 377 \mathrm{Ohms}$ is the impedance of free space. Since the longitudinal electric field depends on the radial position, the LSC impedance also has the same dependence. The LSC impedance per unit length for arbitrary $r$ within the beam $(r<a)$ is given by

$$
\frac{Z_{\|}^{\text {free }}(k, r)}{L}=i \frac{Z_{0}}{k \pi a^{2} \beta}\left[1-\frac{k a}{\gamma} K_{1}\left(\frac{k a}{\gamma}\right) I_{0}\left(\frac{k r}{\gamma}\right)\right] .
$$

Inside a round beam chamber with inner wall radius $r_{w}$, the continuity conditions at $r=a$ and the boundary condition on the chamber surface $E_{z}\left(r=r_{w}\right)=0$ determine the coefficients $A_{1}, A_{2}$ and $A_{3}$ in Eq. (14). Therefore the final solution of the longitudinal electric field is

$$
E_{z}^{r d}(k, r)= \begin{cases}-i \frac{\lambda_{z}}{k \pi a^{2} \varepsilon_{0}}\left\{1-\frac{k a}{\gamma} \frac{I_{0}\left(\frac{k r}{\gamma}\right)}{I_{0}\left(\frac{k r_{w}}{\gamma}\right)}\left[K_{1}\left(\frac{k a}{\gamma}\right) I_{0}\left(\frac{k r_{w}}{\gamma}\right)+K_{0}\left(\frac{k r_{w}}{\gamma}\right) I_{1}\left(\frac{k a}{\gamma}\right)\right]\right\} & (r \leq a), \\ -i \frac{\lambda_{z}}{k \pi a^{2} \varepsilon_{0}} \frac{k a}{\gamma} I_{1}\left(\frac{k a}{\gamma}\right)\left[K_{0}\left(\frac{k r}{\gamma}\right)-\frac{K_{0}\left(\frac{k r_{w}}{\gamma}\right)}{I_{0}\left(\frac{r_{w}}{\gamma}\right)} I_{0}\left(\frac{k r}{\gamma}\right)\right] & \left(a<r \leq r_{w}\right) .\end{cases}
$$


The superscript " $r d$ " on the left-hand side stands for "round chamber." The above equation gives the well-known LSC impedance per unit length of a round uniform beam inside a round beam chamber [13-16]

$$
\frac{Z_{\|}^{r d}(k, r)}{L}=i \frac{Z_{0}}{\mathrm{k} \pi a^{2} \beta}\left\{1-\frac{k a}{\gamma} \frac{I_{0}\left(\frac{k r}{\gamma}\right)}{I_{0}\left(\frac{k r_{w}}{\gamma}\right)}\left[K_{1}\left(\frac{k a}{\gamma}\right) I_{0}\left(\frac{k r_{w}}{\gamma}\right)+K_{0}\left(\frac{k r_{w}}{\gamma}\right) I_{1}\left(\frac{k a}{\gamma}\right)\right]\right\} .
$$

Using the identity of $\left\langle I_{0}(\mathrm{kr} / \gamma)\right\rangle=2 I_{1}(\mathrm{ka} / \gamma) /(\mathrm{ka} / \gamma)$, the averaged LSC impedance over the beam cross section per unit length in free space and inside a round chamber can be derived easily from Eqs. (18) and (20) as

$$
\begin{gathered}
\frac{\bar{Z}_{\|}^{\text {free }}(k)}{L}=i \frac{Z_{0}}{k \pi a^{2} \beta}\left[1-2 K_{1}\left(\frac{k a}{\gamma}\right) I_{1}\left(\frac{k a}{\gamma}\right)\right], \\
\frac{\bar{Z}_{\|}^{r d}(k)}{L}=i \frac{Z_{0}}{\mathrm{k} \pi a^{2} \beta}\left\{1-2 \frac{I_{1}\left(\frac{k a}{\gamma}\right)}{I_{0}\left(\frac{k r_{w}}{\gamma}\right)}\left[K_{1}\left(\frac{k a}{\gamma}\right) I_{0}\left(\frac{k r_{w}}{\gamma}\right)+K_{0}\left(\frac{k r_{w}}{\gamma}\right) I_{1}\left(\frac{k a}{\gamma}\right)\right]\right\} .
\end{gathered}
$$

In the long-wavelength limit $[\gamma /(k a) \gg 1]$, the on-axis LSC impedance of a round beam in free space is

$$
\frac{Z_{\|}^{\mathrm{free}, \mathrm{LW}}(k, 0)}{L}=i \frac{k Z_{0}}{2 \pi \beta \gamma^{2}}\left[\frac{1}{2}-C-\ln \left(\frac{k a}{2 \gamma}\right)\right],
$$

where $C=0.577216$ is the Euler's constant, the superscript "LW" stands for the "long-wavelength limit." The LSC impedance of a round beam centered inside a round beam pipe in the long-wavelength limit is [17-21]

$$
\frac{Z_{\|}^{r d, \mathrm{LW}}(k)}{L}=i \frac{Z_{0} k}{2 \pi \beta \gamma^{2}}\left[C_{1}+\ln \left(\frac{r_{w}}{a}\right)\right],
$$

where $C_{1}=1 / 2$ and $1 / 4$ for the on-axis and average impedance, respectively. Figure 1 shows the comparison of

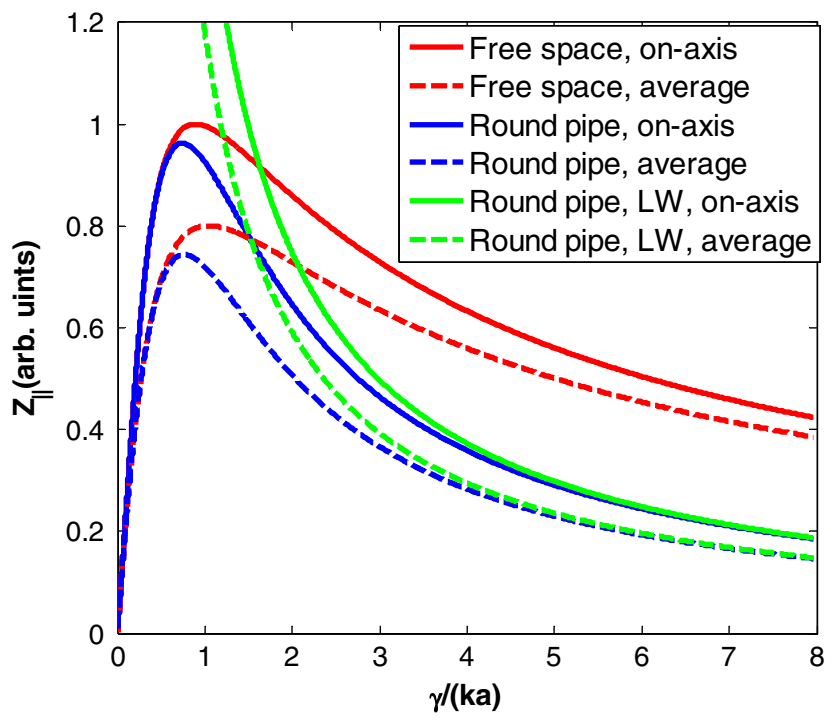

FIG. 1. Comparison of the on-axis (solid lines) and average (dashed lines) LSC impedances of a round beam in free space and inside a round chamber. In the legend, "LW" stands for the longwavelength limit. The ratio of $r_{w} / a$ is 2 for this example. the LSC impedance of a round beam in free space [Eqs. (18) and (21)] and inside a round chamber [Eqs. (20) and (22)]. Both the on-axis and average impedance are plotted for the purpose of comparison. The shielding of the beam chamber becomes more effective when $\gamma /(k a)>1$. The formula of LSC impedance in the long-wavelength limit [(Eq. (24)] works well only when $\gamma /(k a) \gg 1$. Therefore, derivation of a full-spectrum analytical LSC impedance formula becomes necessary and important, which is one of the motivations of this paper.

\section{BETWEEN PARALLEL PLATES}

Figure 2 shows the cross sections of a source beam between a pair of parallel plates and its images. We apply the image method by disregarding the boundary at each plate in a stepwise fashion to create the image map. Assuming the source beam has a line charge density of

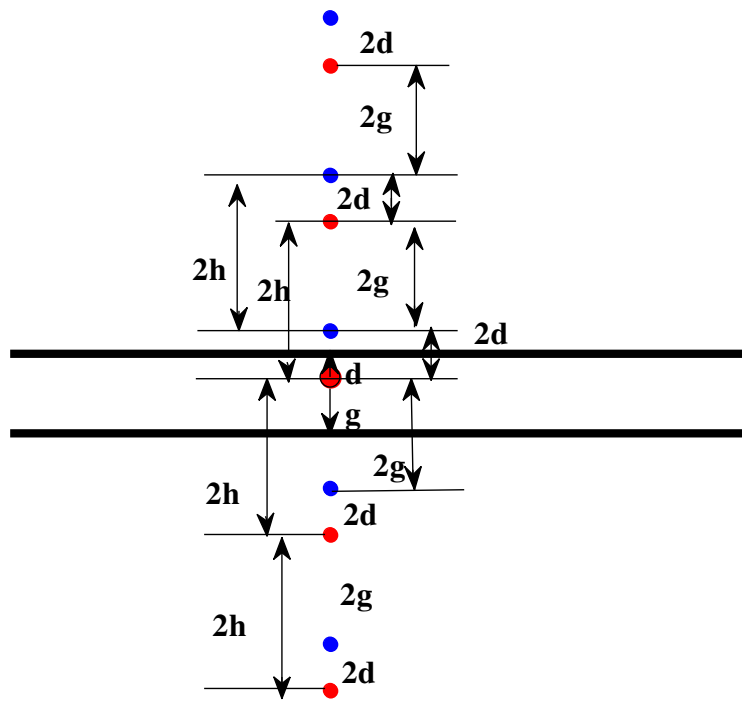

FIG. 2. Sequence of images of a source beam with vertical offset between a pair of parallel plates. 
$\Lambda(z)=\lambda_{\mathrm{z}} \cos (k z)$, the red and blue dots represent the beams with line charge densities of $\Lambda(z)$ and $-\Lambda(z)$, respectively. The distances of the source beam axis to the upper and lower plates are $d$ and $g$, respectively. The full separation between the two plates is $h=g+d$. If the source beam axis is located on the median plane between the two plates, then we have $g=d=h / 2$.

Let us assume that the median plane of the parallel plate has vertical coordinate $y=0$ and a source beam is located at $\left(0, y_{c}\right)$, as shown in Fig. 2 . The image beams have the vertical coordinates of $y_{\mathrm{img}}(n)=n h+(-1)^{n} y_{c}$ and the corresponding line charge densities amplitudes of $\lambda_{z \text {,imag }}(n)=(-1)^{n} \lambda_{z}, n=0, \pm 1, \pm 2 \ldots$. The images with the index of $n>0$ and $n<0$ represent the ones above and below the parallel plates, respectively. The term with index of $n=0$ corresponds to the original source beam. For a round beam with uniform transverse density, its image beams have the same density distribution as the source beam as shown in Fig. 3. Note that the image beams within an arbitrary chamber may not always have the same shape as the source beam, for instance, the image beams within an elliptical chamber.

The field at any field point is equal to the sum of all image fields plus the self-field of the original source beam in free space. For instance, the LSC field at position $(x, y)$ within the source beam is

$$
E_{z}^{p p}(k, x, y)=\sum_{n=-\infty}^{\infty}(-1)^{n} E_{z}^{\text {free }}\left(k, R_{n}\right)
$$

where $R_{n}=\sqrt{x^{2}+\left(y_{\text {img }}(n)-y\right)^{2}}$. The superscript " $p p$ " on the left hand of Eq. (25) stands for "parallel plates." The term with $n=0$ on the right-hand side of Eq. (25) is contributed from the original source beam. Using Eqs. (8), (15) and (25), we obtain the LSC impedance per unit length at position $(x, y)$ inside the beam as

$$
\frac{Z_{\|}^{p p}(k, x, y)}{L}=i \frac{Z_{0}}{\mathrm{k} \pi a^{2} \beta}\left[1-\frac{k a}{\gamma} K_{1}\left(\frac{k a}{\gamma}\right) I_{0}\left(\frac{k R_{0}}{\gamma}\right)+\frac{k a}{\gamma} I_{1}\left(\frac{k a}{\gamma}\right) \sum_{\substack{n=-\infty \\ n \neq 0}}^{\infty}(-1)^{n} K_{0}\left(\frac{k R_{n}}{\gamma}\right)\right] .
$$

Different from the free space case, the LSC field of a round beam between parallel plates is not axisymmetric due to the boundary shape. Therefore, the impedance is a function of $(x, y)$ instead of $r$ exclusively.

Figure 4 shows an example of the calculated on-axis impedance with different numbers of image beams. The full height of the parallel plate is $h=4 a$ in this example. The image beams affect the impedance mostly in the longwavelength regime where $\gamma /(k a)>1$. This clearly explains why the shielding is effective only for the long wavelength perturbations. The impedance with $|n|=1$ has the smallest value because it includes only the first pair of image beams whose line charge densities are opposite to

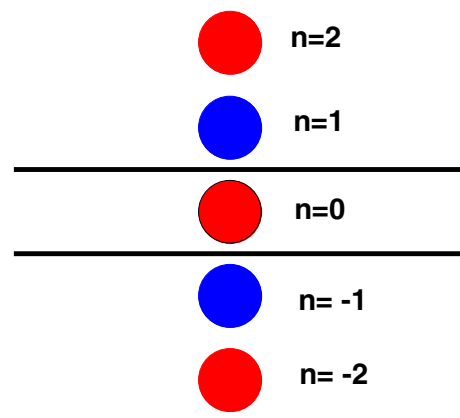

FIG. 3. Sequence of images of an infinitely long and uniform round beam centered between a pair of parallel plates. The image beams $(n \neq 0)$ have the same shape and dimension as the source beam $(n=0)$. that of the original beam. On the other hand, the impedance with $|n|=2$ has the maximum value. Similarly, $|n|=3$ gives the second smallest one and $|n|=4$ gives the second

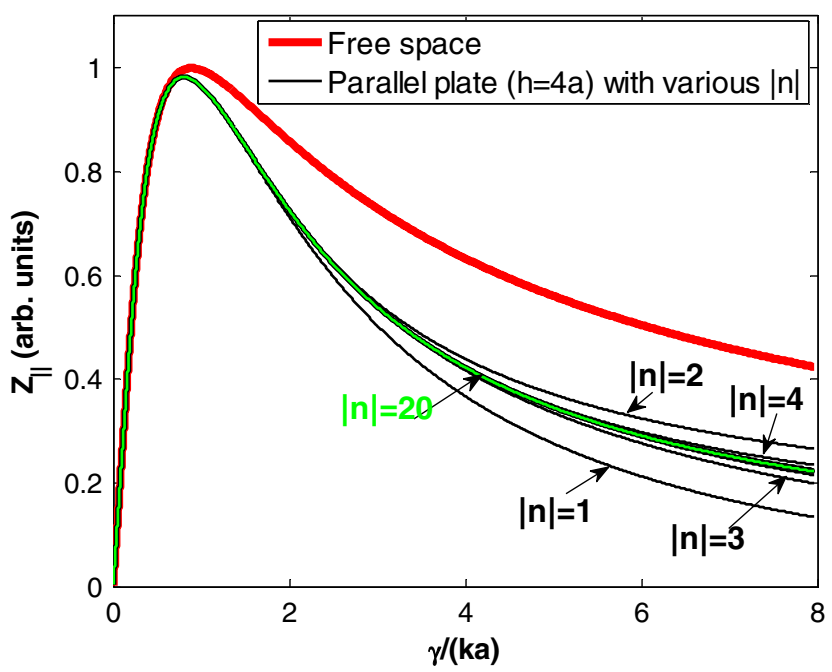

FIG. 4. The effect of image bunches on the on-axis LSC impedance of a round transversely uniform beam centered between a parallel-plate chamber. The bunch has a radius of $a=5 \mathrm{~mm}$. The full height of the parallel plate is $h=4 a=20 \mathrm{~mm}$. The family of black lines shows the calculated impedances with different maximum index $|n|$ of image beams in Eq. (26) ranging from 1 to 20 . The green line in the plot shows the calculated impedance with $|n|=20$. 


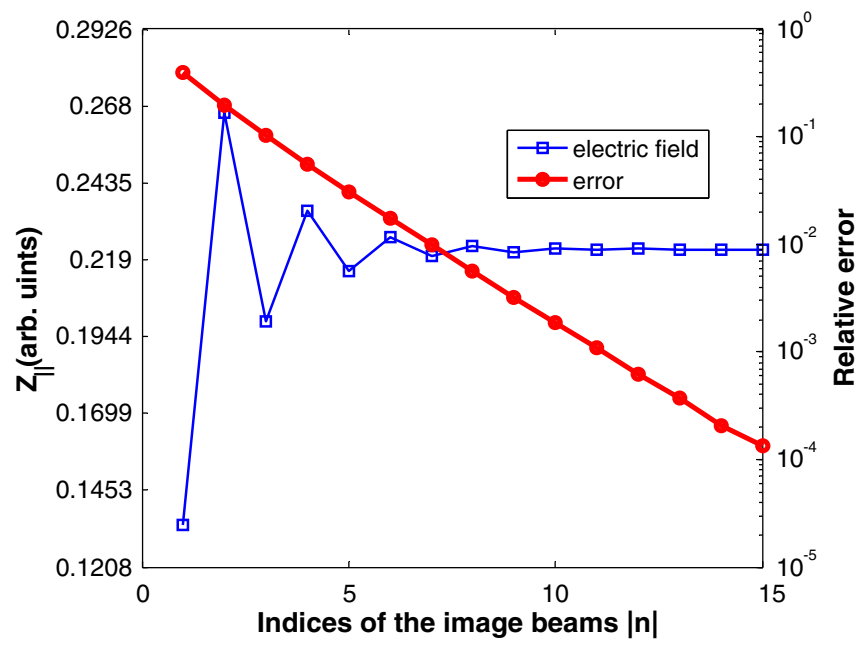

FIG. 5. Convergence of the on-axis LSC impedance with the maximum index of image beams for a parallel-plate chamber. The bunch has a radius of $a=5 \mathrm{~mm}$ and the full separation of the parallel plate is $h=4 a=20 \mathrm{~mm}$. This result corresponds to the case $\gamma /(k a)=8$ as shown in Fig. 4 where the image charge effect is relatively larger.

largest one and so on. When $|n|$ is large enough, the variation of the impedance is negligible because the image beams are far away from the source beam and the field finally converges.

Figure 5 shows the variation of the on-axis LSC impedance at $\gamma /(k a)=8$ with the maximum index $|n|$ of image beams $(h / a=4)$. The calculated impedance (blue curve) oscillates at small $|n|$ and converges to one value at large $|n|$. The relative errors (red curve) in the plot are the absolute values of the relative errors. The true errors oscillate with $|n|$ and have negative sign for odd numbers of $|n|$. The absolute magnitudes of the relative errors decay exponentially which implies that the field converges with $|n|$ quickly. The case of $|n|=15$ gives a relative error in the order of magnitude of $10^{-4}$.

When the beam axis has vertical offsets with respect to the chamber median plane (see Fig. 2), the on-axis LSC impedance at long wavelength is slightly reduced as shown in Fig. 6, assuming $h=10 a$ and the beam offsets range from $a$ to $4 a$. The beam offset only affects the longwavelength impedance for the same mechanism as the shielding effect. When the beam axis shifts vertically from the chamber median plane, one group of images with line charge densities of $-\Lambda(z)$ (with $y_{\mathrm{img}}>0$ in Fig. 2) moves closer to the beam while another group of images with line charge densities of $-\Lambda(z)$ (with $y_{\text {img }}<0$ in Fig. 2) moves away from it, as shown in Fig. 2. The distance of the image beams with line charge densities of $\Lambda(z)$ to the source beam does not change when the source beam is shifted. The net effect from the redistribution of the image beams with line charge densities of $-\Lambda(z)$ is small due to the cancellations of all the image beams. In general, the effect of beam offset

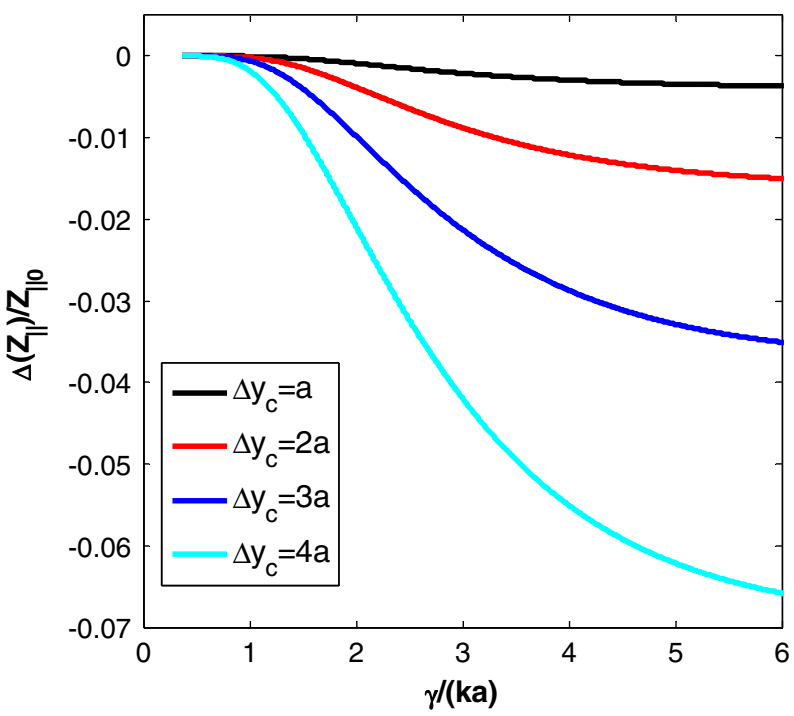

FIG. 6. Effect of vertical beam axis offset $\left(\Delta y_{c}\right)$ on the on-axis LSC impedance of a parallel-plate chamber with $h=10 a$. The relative impedance difference $\Delta Z_{\|}$is normalized by the on-axis LSC impedance $Z_{\| 0}$ with $\Delta y_{c}=0$. The impedance at long wavelength is slightly reduced when the beam axis has small vertical offsets.

is negligible if the offset amplitude is small compared to the aperture of beam pipe.

The LSC impedance of a round beam centered between parallel plates in the long-wavelength limit can be derived from Ref. [22] as

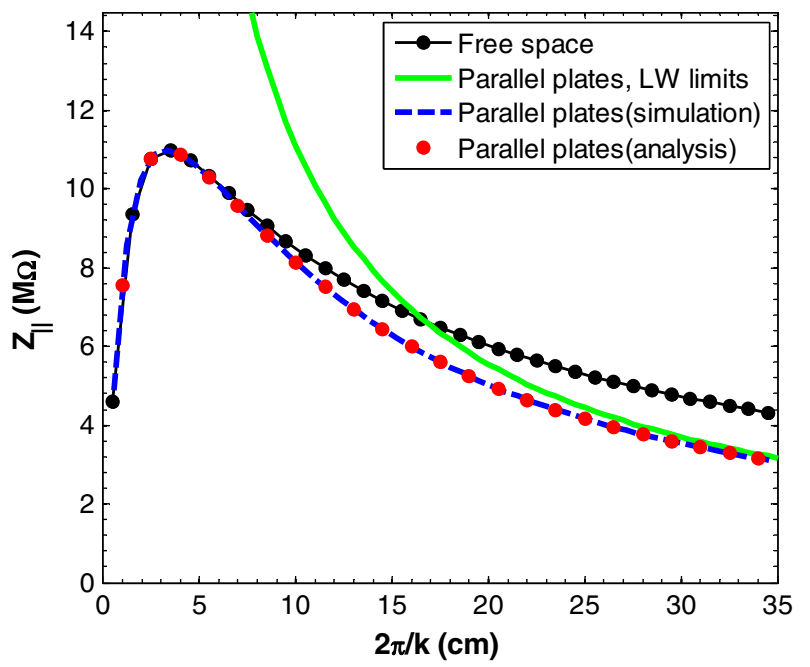

FIG. 7. Comparison of the average LSC impedances of a round SIR beam with beam radius $a=0.5 \mathrm{~cm}$ under different boundary conditions and in different wavelength limit. The full height of the parallel plates is $4.8 \mathrm{~cm}$. In the legend, "Free space", and "Parallel plates" are boundary conditions; "LW limits" stands for the long-wavelength limits; "(analysis)" and "(simulation)" stand for the theoretical result and simulation (FEM) method, respectively. The theoretical result is consistent with the simulation in the entire wavelength spectrum. 


$$
\frac{Z_{\|}^{p p, \mathrm{LW}}(k)}{L}=i \frac{Z_{0} k}{2 \pi \beta \gamma^{2}}\left[C_{1}+\ln \left(\frac{2 h}{\pi a}\right)\right],
$$

where $C_{1}=1 / 2$ for the on-axis impedance and $C_{1}=1 / 4$ for the average one, respectively. Figure 7 shows the comparison of the average LSC impedances of a round beam with beam radius $a=0.5 \mathrm{~cm}$ under different boundary conditions and in a different wavelength limit. We used the $20 \mathrm{keV}(\beta \approx 0.0046, \gamma \approx 1.0)$ coasting $\mathrm{H}_{2}{ }^{+}$beam in the small isochronous ring (SIR) at Michigan State University (MSU) [23] in this example. The circumference of SIR is $6.58 \mathrm{~m}$. The analysis agrees well with the simulation in the entire wavelength spectrum; while the impedance formula of the long-wavelength limit works well only in the case of $\gamma /(k a)>11.14$ (with the charge density perturbation wavelength $2 \pi / k=35 \mathrm{~cm}$ in Fig. 7).

\section{INSIDE A RECTANGULAR CHAMBER}

Consider an infinitely long, transversely uniform round beam inside a rectangular conducting structure with full width $w$ and full height $h$. We assume the axes of the rectangular chamber and the beam are located at $(0,0)$ and $\left(x_{c}, y_{c}\right)$, respectively. Figure 8 shows the image beams of a source beam centered inside a rectangular chamber indicated by the solid black rectangle. The red and blue dots represent the beams with line charge densities of $\Lambda(z)$ and $-\Lambda(z)$, respectively.

Through planar symmetry and mirroring, we can determine the exact coordinates of images axes of a line beam inside a rectangular chamber as

$$
\begin{aligned}
x_{\text {img }}(m) & =m w+(-1)^{m} x_{c}, \\
y_{\text {img }}(n) & =n h+(-1)^{n} y_{c}, \\
\lambda_{z, \text { imag }}(m, n)=(-1)^{m+n} \lambda_{z}, m & =n=0, \pm 1,2,3 \ldots
\end{aligned}
$$

Here $m$ and $n$ represent the index of the image grid points in horizontal and vertical directions, respectively. For instance, the index $m>0(m<0)$ is for the images with $x>0(x<0)$. The image with the index of $m=n=0$ is just the original source beam. Similar to the case of parallelplate chamber, the image beams of a round uniform beam still keep the same distributions and beam radius as those of the source beam, while their centers are given by Eq. (28). Therefore, the LSC field at $(x, y)$ within the source beam inside a rectangular chamber is equal to the total LSC fields of the source beam and its image beams in free space,

$$
E_{z}^{\mathrm{rect}}(k, x, y)=\sum_{m=-\infty}^{\infty} \sum_{n=-\infty}^{\infty}(-1)^{m+n} E_{z}^{\mathrm{free}}\left(k, R_{m, n}\right),
$$

with $\quad R_{m, n}=\sqrt{\left(x_{\text {img }}(m)-x\right)^{2}+\left(y_{\text {img }}(n)-y\right)^{2}}$. The superscript "rect" on the left hand of the equation stands for "rectangular chamber." Note that the original source beam effect is included with $m=n=0$. Using Eqs. (8), (15) and (29), we obtain the LSC impedance per unit length at position $(x, y)$ inside the beam as

$$
\frac{Z_{\|}^{\text {rect }}(k, x, y)}{L}=i \frac{Z_{0}}{\mathrm{k} \pi a^{2} \beta}\left[1-\frac{k a}{\gamma} K_{1}\left(\frac{k a}{\gamma}\right) I_{0}\left(\frac{k R_{0,0}}{\gamma}\right)+\frac{k a}{\gamma} I_{1}\left(\frac{k a}{\gamma}\right) \sum_{m=-\infty}^{m=\infty} \sum_{\substack{m \neq 0 \\ m \neq 0}}^{n=\infty}(-1)^{m+n} K_{0}\left(\frac{k R_{m, n}}{\gamma}\right)\right] .
$$

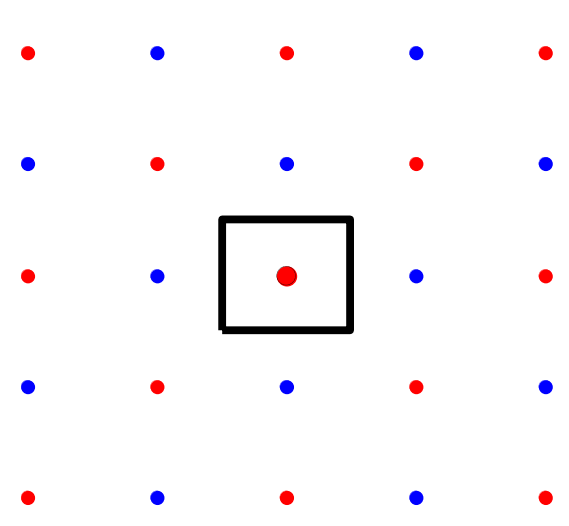

FIG. 8. 2D grid of images of a source beam centered inside a rectangular chamber.
The LSC impedance per unit length of a round uniform beam centered inside a rectangular chamber in the longwavelength limit is [7]

$$
\frac{Z_{\|}^{\text {rect, } \mathrm{LW}}(k)}{L} \approx i \frac{Z_{0} k}{2 \pi \beta \gamma^{2}}\left\{C_{1}+\ln \left[\frac{2 h}{\pi a} \tanh \left(\frac{\pi w}{2 h}\right)\right]\right\}, \quad \frac{w}{h}>1,
$$

where $C_{1}=1 / 2$ for the on-axis impedance and $C_{1}=1 / 4$ for the average one, respectively. The above formula is a good approximation for a rectangular chamber with $w / h>1$. When $w / h$ is about 1 , the exact but more complicated formula [7] should be used. 


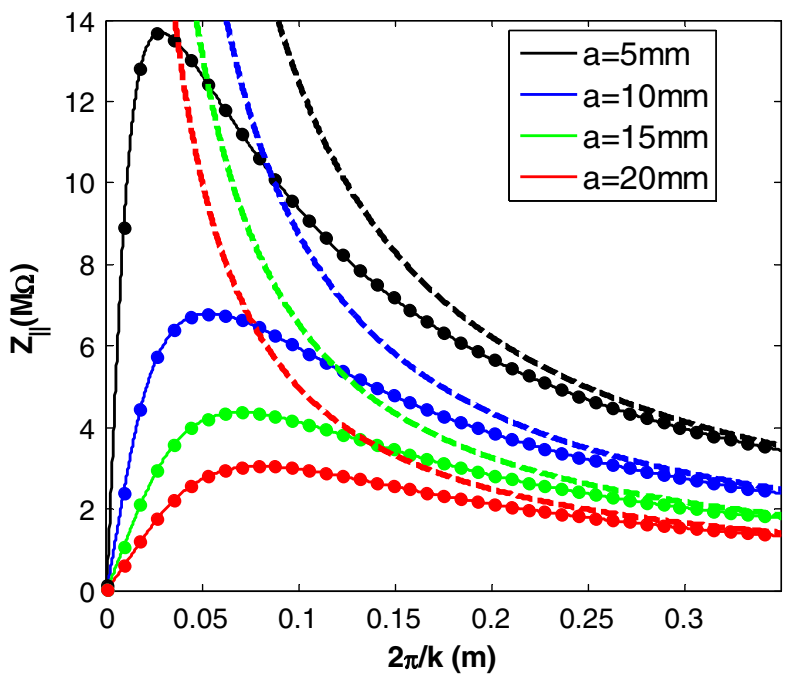

FIG. 9. Comparison of the on-axis LSC impedances of a round transversely uniform beam centered inside the SIR rectangular chamber between analysis (solid lines) and FEM simulations (solid dots). The LSC impedances predicted by the longwavelength-limit formula (dashed lines) are also shown for comparison. The parameters used in the calculations are $w=11.4 \mathrm{~cm}, h=4.8 \mathrm{~cm}$, the variable beam radii are $a=0.5$, $1.0,1.5$ and $2.0 \mathrm{~cm}$ in this study. The circumference of SIR is $6.58 \mathrm{~m}$.

Figure 9 shows the comparison of the on-axis LSC impedances obtained by the theoretical calculations and the simulations using a finite element method (FEM) code. We used the $20 \mathrm{keV}$ SIR beam in this example. The cross section of the SIR chamber is rectangular with $w=$ $11.4 \mathrm{~cm}$ and $h=4.8 \mathrm{~cm}$. The maximum index of the image beam used in the calculation is $|m|=|n|=10$. The on-axis LSC impedances of the beam with four different beam radii are compared to each other. The analytical results (solid lines) and FEM results (circles) perfectly overlap each other demonstrating excellent agreements in all cases in the entire wavelength spectrum. However, the long-wavelength-limit formula significantly overestimates the impedance at short wavelength as shown by the dashed lines in Fig. 9. Therefore, it is essential to use a more accurate full-spectrum LSC impedance formula in the study of beam instability.

Figure 10 shows the shielding effect of a square chamber with $w=h=4 a, 6 a, 8 a, \ldots 16 a$. The shielding effect is negligible at short wavelengths with $\gamma / k a<1.0$, but it becomes more noticeable at long wavelengths. For a fixed beam radius, the shielding effect becomes weaker as the chamber aperture is enlarged because the image beams are farther away from the source beam.

Figure 11 shows the effect of beam offset on the on-axis impedance in a square chamber with $w=h=10 a$. The beam offset reduces the impedance and this reduction depends on the wavelength as shown in Fig. 11(a) where the vertical offset is zero. The beam offset effectively

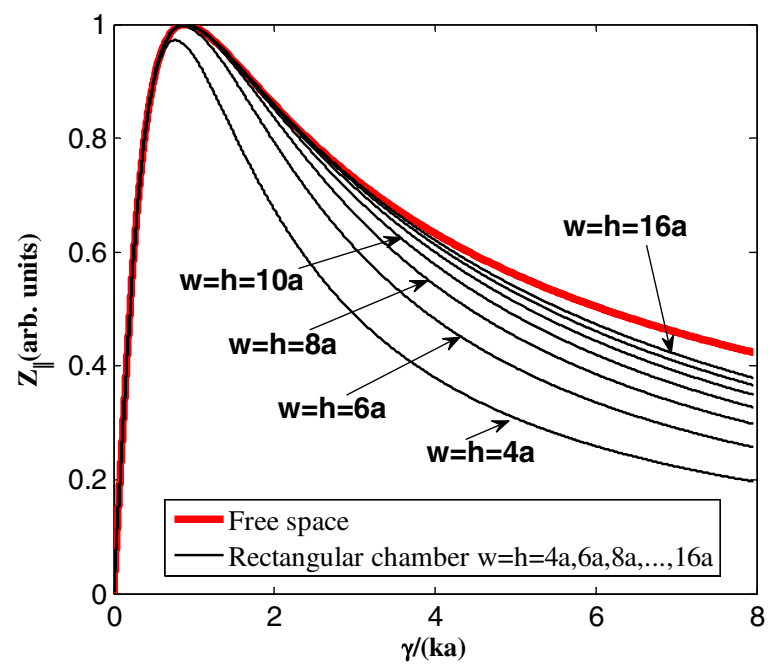

FIG. 10. The shielding effect of a rectangular chamber on the on-axis impedance. The round uniform beam has a radius of $a$, the full widths and heights of the chamber are $w=h=4 a, 6 a$, $8 a, 10 a, 12 a, 14 a$ and $16 a$, which correspond to the lower to upper black lines as clearly shown on the right part of the plot.

reduces the impedance at a long-wavelength regime due to the chamber shielding effect. Figure 11(b) presents a particular wavelength with $\gamma /(k a)=5$. The reduction of the LSC impedance is small when the beam offset is much smaller when compared to the aperture of the beam pipe. However, the reduction of the impedance becomes more pronounced when the beam offset increases because of the shielding effect by the image beams, which roughly scales as $1 / r$ ( $r$ is the distance of the source beam axis to the surface of beam pipe). The impedance is about $50 \%$ smaller when the beam is close to the surface of the beam pipe as shown in the figure.

Figure 12 compares the shielding effect on the on-axis impedance between a round chamber $\left(r_{w}=2 a\right)$, parallel plates $(h=4 a)$ and rectangular chambers $(h=4 a$, $w / h=1$ and 2). The on-axis LSC impedance in free space and inside a round chamber is calculated using Eqs. (17) and (20) for $r=0$, respectively. The round chamber has slightly stronger shielding than a square chamber resulting in a smaller on-axis LSC impedance. It is about 6\% (1\%) less than that of a round beam inside a square pipe when the pipe radius is $2 a(10 a)$ at wavelength regime with $8>\gamma /(k a)>2$. When the aspect ratio of rectangular chamber $w / h$ is larger than 2 , the shielding effect is very close to that of a parallel-plate model. The impedance of a parallel-plate model at long-wavelength regime is about $20 \%$ larger than that of the round-chamber model.

To compare the shielding effect in the long-wavelength limit we can define the geometry factor $g_{0}$ as

$$
\frac{Z_{\|}^{\mathrm{LW}}(k)}{L}=i \frac{Z_{0} k}{2 \pi \beta \gamma^{2}} g_{0}
$$



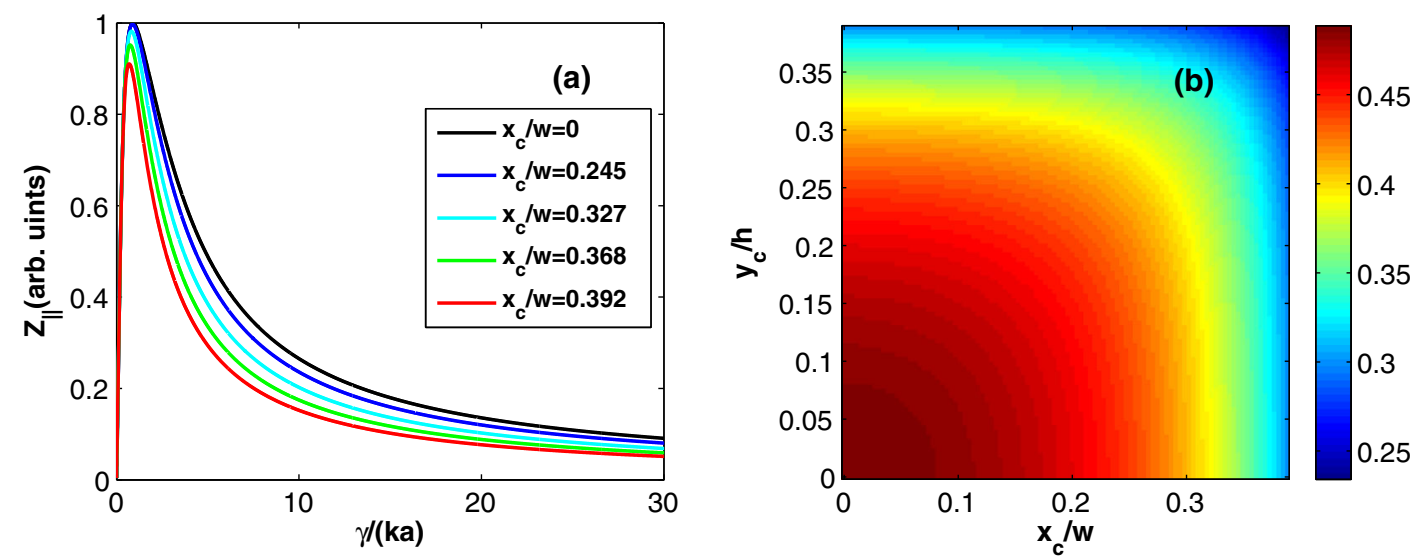

FIG. 11. The effect of beam offset $\left(x_{c}, y_{c}\right)$ in a square chamber on the on-axis impedance. The impedance in the entire wavelength spectrum with different horizontal offsets $x_{c}\left(y_{c}=0\right)$ is shown in plot (a); and the impedance at one particular wavelength $\gamma /(k a)=5$ for various $\left(x_{c}, y_{c}\right)$ is shown in (b). The round uniform beam has a radius of $a$; the full width and height of the chamber are $w=h=10 a$.

Comparing Eqs. (24), (27) and (31) with the above equation, we can get the geometry factor $g_{0}$ of a transversely uniform round beam centered inside the round chamber, parallel plates and rectangular chamber as

$g_{0}= \begin{cases}C_{1}+\ln \left(\frac{r_{w}}{a}\right), & \text { round chamber } \\ C_{1}+\ln \left(\frac{2 h}{\pi a}\right), & \text { parallel plates } \\ C_{1}+\ln \left[\frac{2 h}{\pi a} \tanh \left(\frac{\pi w}{2 h}\right)\right], & \text { rectangular chamber, } \frac{w}{h}>1 .\end{cases}$

where $C_{1}=1 / 2$ for the on-axis impedance and $C_{1}=1 / 4$ for the average one, respectively.

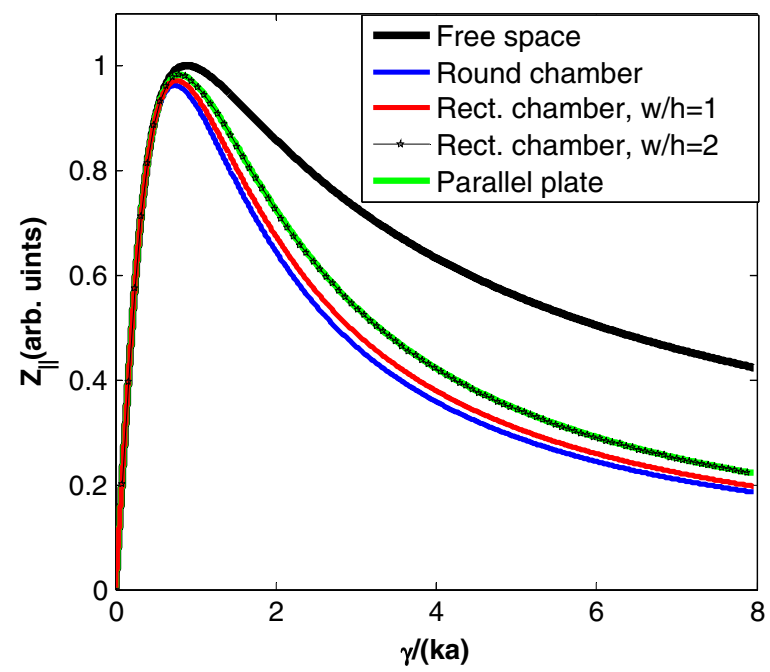

FIG. 12. Comparison of the shielding effect on the on-axis impedance between a round chamber $\left(r_{w}=2 a\right)$, parallel plates ( $\left.h=2 r_{w}=4 a\right)$ and a rectangular chamber $\left(h=2 r_{w}\right)$ with the ratio $w / h=1,2$. The full height of the rectangular chamber $h$ is fixed and equal to the diameter of the round chamber.
Our method can also give the LSC impedances averaged over the beam cross section. Figure 13 shows the average LSC impedance of the round beam with four different boundary conditions: parallel plates, square chamber, round chamber [Eq. (22)], and in free space [Eq. (21)]. The same beam parameters and normalization method are used as in Fig. 12, where the normalized peak impedance in free space is set to 1 . The shielding effect on the average LSC impedances is similar to the case of on-axis LSC impedances. The average LSC impedance of the round beam is about $20 \%$ less than the on-axis one when $1<\gamma /(k a)<8.0$. However, they are almost identical for high frequency with $\gamma /(k a)<1.0$.

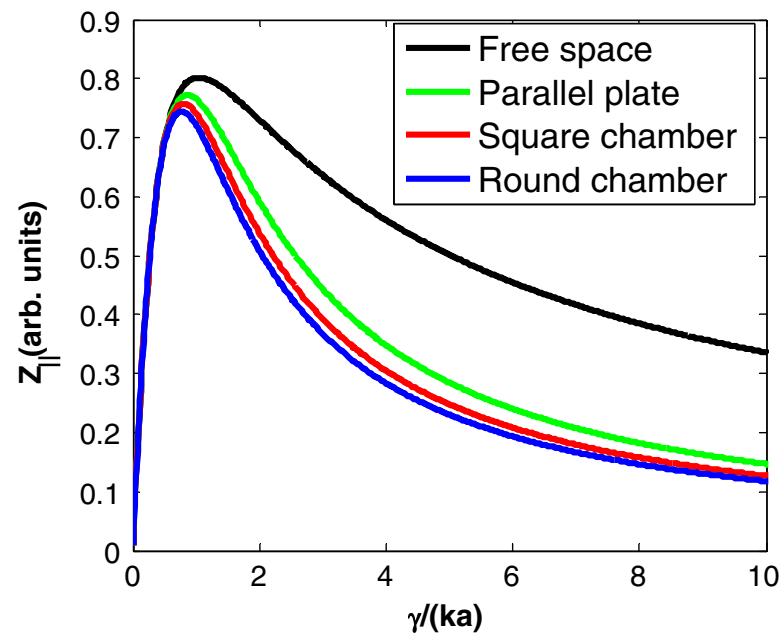

FIG. 13. Comparison of the shielding effect between the round, square, and parallel-plate chambers on the average LSC impedance. The same beam and chamber parameters in Fig. 12 are used here. The square chamber has an aspect ratio of $w / h=1$. 


\section{CONCLUSIONS}

The image charge method is employed in this paper to provide analytical solutions to the full-spectrum LSC impedances of a round uniform beam with line charge modulation inside a rectangular chamber and between parallel plates, respectively. Though the solutions consist of contributions from an infinite number of image beams, due to the fast convergence with the number of image beams, only a finite and small number of image beams are needed to get a satisfying resolution. Since the solutions to the image charge fields induced by each constituent image beam are exact and expressed in a closed form, the resolution of the total calculated LSC fields and impedances is only dependent on the number of image beams used in the calculation and is insensitive to the ratios of transverse dimensions between the chamber and beam. This is a general method applicable to other beam distributions, such as Gaussian, rectangular and elliptic beams. The calculated LSC impedances in our analysis are valid in the full wavelength spectrum and at arbitrary position within the beam. Moreover, the effect of beam axis offset is also included in our analysis.

Our studies show that round chambers have a slightly better shielding effect than square chambers. When the aspect ratio of a rectangular chamber is larger than 2 , the shielding effect is very close to that of a pair of parallel plates. The offset of beam axis slightly reduces the LSC impedance when the offset is small compared to the aperture of beam chamber. However, the reduction becomes significant when the beam is close to the surface of beam chamber.

\section{ACKNOWLEDGMENTS}

L. Wang would like to thank Professor Zhirong Huang at SLAC for fruitful discussions. This work is supported by Department of Energy Contract No. DE-AC0276 SF00515.

[1] E. Pozdeyev, J. A. Rodriguez, F. Marti, and R. C. York, Phys. Rev. ST Accel. Beams 12, 054202 (2009).

[2] Y. Li and L. Wang, Nucl. Instrum. Methods Phys. Res., Sect. A 763, 674 (2014).
[3] Z. Huang, M. Borland, P. Emma, J. Wu, C. Limborg, G. Stupakov, and J. Welch, Phys. Rev. ST Accel. Beams 7, 074401 (2004).

[4] D. Ratner, C. Behrens, Y. Ding, Z. Huang, P. Krejcik, A. Marinelli, and T. Maxwell, The 6th Microbunching Workshop, Trieste, Italy, 2014, https://www.elettra.trieste .it/Conferences/2014/MBIW6/uploads/Main/2014_ubi _Maxwell.pdf.

[5] P. Emma et al., Nat. Photonics 4, 641 (2010).

[6] A. Marinelli, E. Hemsing, M. Dunning, D. Xiang, S. Weathersby, F. O'Shea, I. Gadjev, C. Hast, and J. B. Rosenzweig, Phys. Rev. Lett. 110, 264802 (2013).

[7] K. Y. Ng, Part. Accel. 16, 63 (1984).

[8] J. Rosenzweig, C. Pellegrini, L. Serafini, C. Ternienden, and G. Travish, TESLA FEL Report No. 1996-15.

[9] S. Humphries, Jr., Charged Particle Beams (Wiley, New York, 1990), p. 704.

[10] R. D. Ryne, http://arxiv.org/abs/1111.4971.

[11] Y. Li and L. Wang, Nucl. Instrum. Methods Phys. Res., Sect. A 747, 30 (2014).

[12] Y. Li and L. Wang, Nucl. Instrum. Methods Phys. Res., Sect. A 769, 44 (2015).

[13] K. Y. Ng, Physics of Particle Accelerators, AIP Conf. Proc. No. 184, edited by M. Month and M. Dienes (AIP, New York, 1989), Vol. 1, p. 472.

[14] H. Hahn, Part. Accel. 51, 181 (1995).

[15] R. L. Gluckstern, CERN Report No. 2000-011, 2000.

[16] A. M. Al-khateeb, O. Boine-Frankenheim, R. W. Hasse, and I. Hofmann, Phys. Rev. E 71, 026501 (2005).

[17] V. K. Neil and A. M. Sessler, Rev. Sci. Instrum. 36, 429 (1965).

[18] R. L. Gluckstern, J. vanZeijts, and B. Zotter, Phys. Rev. E 47, 656 (1993).

[19] F. Ruggiero, Part. Accel. 50, 83 (1995).

[20] A. W. Chao, Physics of Collective Beam Instabilities in High Energy Accelerators (John Wiley \& Sons, Inc., New York, 1993), pp. 68-71.

[21] M. Venturini, Phys. Rev. ST Accel. Beams 11, 034401 (2008).

[22] C. E. Nelson and A. M. Sessler, Rev. Sci. Instrum. 30, 80 (1959).

[23] E. Pozdeyev, Ph.D. thesis, Michigan State University, 2003.

[24] M. A. Furman, Phys. Rev. ST Accel. Beams 10, 081001 (2007).

[25] See e.g., J. N. Reddy, An Introduction to the Finite Element Method, 3rd ed. (McGraw-Hill, New York, 2006).

[26] J. D. Jackson, Classical Electrodynamics, 2nd ed. (Wiley New York, 1975). 